CORRIGENDUM

doi:10.1038/nature 12317

\title{
Corrigendum: Glutamine supports pancreatic cancer growth through a KRAS-regulated metabolic pathway
}

Jaekyoung Son, Costas A. Lyssiotis, Haoqiang Ying,

Xiaoxu Wang, Sujun Hua, Matteo Ligorio, Rushika M. Perera, Cristina R. Ferrone, Edouard Mullarky, Ng Shyh-Chang, Ya'an Kang, Jason B. Fleming, Nabeel Bardeesy, John M. Asara, Marcia C. Haigis, Ronald A. DePinho, Lewis C. Cantley

\& Alec C. Kimmelman

Nature 496, 101-105 (2013); doi:10.1038/nature12040

In the Supplementary Information of this Letter, four images were inadvertently duplicated. This error has no impact on the data presented in the Letter because they were representative images of clonogenic assays; the primary data presented in the main text and figures are unchanged. In Supplementary Fig. 1b, the second plate from the right was a duplicate of the third plate from the right in Supplementary Fig. 1b. In Supplementary Fig. 12, the second plate in the top row was a duplicate of the first plate in Supplementary Fig. 2b. In Supplementary Fig. 14b, the bottom right plate was a duplicate of the second plate in the third row of Supplementary Fig. 14c. In Supplementary Fig. 18c, the last plate of the top row was a duplicate of the first plate in Supplementary Fig. 2b. In the Supplementary Information to this Corrigendum we now provide the corrected panels for Supplementary Figs 1b, 12, 14b and 18c.

In addition, Supplementary Figs $11 \mathrm{~b}$ and $14 \mathrm{~b}$ were derived from the same experiment and share a panel: the first plate of Supplementary Fig. $11 \mathrm{~b}$ is the same as the first plate in the first row of Supplementary Fig. 14b. Similarly, the first plate in the second row of Supplementary Fig. 13b is the same as the first plate in the second row of Supplementary Fig. 14a; the last plate in the second row of Supplementary Fig. 13b is the same as the second plate in the second row of Supplementary Fig. 14a; the first plate in the third row of Supplementary Fig. $13 \mathrm{~b}$ is the same as the third plate in the second row of Supplementary Fig. 14a; and the last plate in the third row of Supplementary Fig. 13b is the same as the last plate in the second row of Supplementary Fig. 14a. No changes to these figures are necessary; we simply point this out for clarification.

Supplementary Information is available in the online version of this Corrigendum. 